\title{
Heartbeat: Early cardiomyopathy or physiological left ventricular dilation in athletes?
}

doi:10.1136/heartjnl-2020-317514

Catherine M Otto

Athletic physical conditioning results in changes in cardiac anatomy and function that make it challenging to distinguish normal physiological changes from a pathological process in athletes with left ventricular (LV) dilation or a reduced ejection fraction. Millar and colleagues ${ }^{1}$ propose an approach for identifying athletes with dilated cardiomyopathy (DCM) based on sequential evaluation using N-terminal probrain natriuretic peptide (NT-proBNP) measurement, resting ECG, ambulatory ECG monitoring, exercise echocardiography and cardiac magnetic resonance (CMR) imaging. In 25 male athletes with LV dilation and an ejection fraction $<55 \%$ compared with 35 asymptomatic active male patients with DCM, a failure to increase ejection fraction $>11 \%$ compared with baseline on exercise echocardiography had the highest sensitivity, with a high specificity, for prediction of DCM. (figure 1)

Donal, Paven and Galli ${ }^{2}$ comment that: "In clinical practice, when a physician does not understand the patient's symptoms on effort, he might suggest an exercise test. Combining exercise and imaging, we can obtain a comprehensive information on heart anatomy and function, which might improve our understanding of the disease, its symptoms and prognosis. Increase in pathophysiological understanding might provide further insight to dealing with symptoms and eventually propose more specific treatments (figure 2). More work has to be done. But frankly Millar et al's ${ }^{1}$ work is an important contribution to promoting exercise stress echocardiography for practical clinical reasoning."

Another interesting paper in this issue of Heart by Jepma and colleagues ${ }^{3}$ compared the effect of a community-based lifestyle intervention programme in older (age 65 years or older) vs younger (age $<65$ years) patients with coronary artery disease. Perhaps surprisingly, despite a higher prevalence of adverse cardiovascular risk factors and comorbidities, older patients were over $5 \mathrm{X}$ more likely to achieve weight loss of $5 \%$ or greater, whereas younger patients were more likely to show

Correspondence to Professor Catherine M Otto, Division of Cardiology, University of Washington, Seattle, WA 98115, USA; cmotto@uw.edu

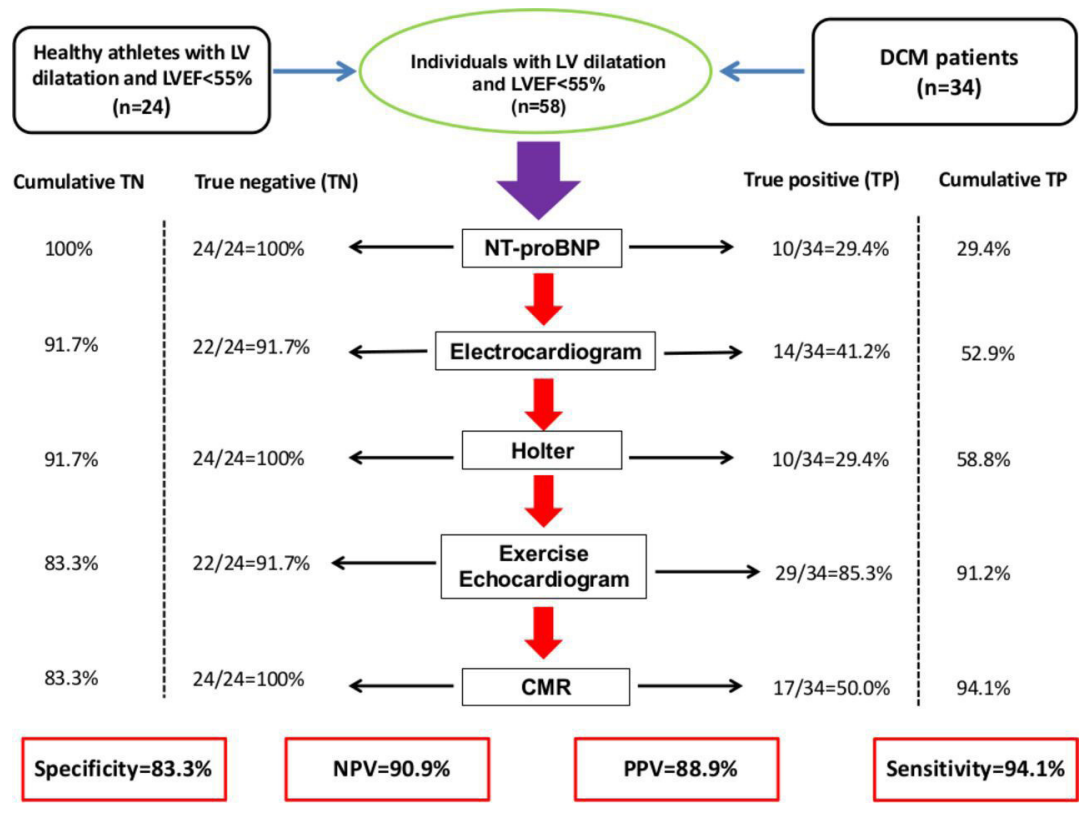

Figure 1 The figure demonstrates the utility of the stepwise clinical algorithm for differentiating between physiological adaptation and morphologically mild DCM in apparently healthy individuals with LV dilatation and LVEF $<55 \%$. The number and percentages of both cohorts with abnormal investigations is shown with the cumulative TN and TP results on the extreme right and left, respectively. The overall sensitivity of the algorithm is $94.1 \%$ with a specificity of $83.3 \%$. The PPV is $90.3 \%$ with an NPV of $94.7 \%$. CMR, cardiovascular magnetic resonance; DCM, dilated cardiomyopathy; LV, left ventricular; LVEF, left ventricular ejection fraction; NPV, negative predictive value; NT-proBNP, N-terminal pro-brain natriuretic peptide; PPV, positive predictive value; TN, true negative; TP, true positive.

no improvement in life-style related risk factors (physical activity, weight reduction, smoking cessation). This findings highlight the important of lifestyle-interventions in older adults with coronary disease both in

terms of each patient's quality of life but also in terms of avoiding future adverse events and reducing healthcare costs.

In an editorial, Norekvål and Allore ${ }^{4}$ point out that cardiac rehabilitation

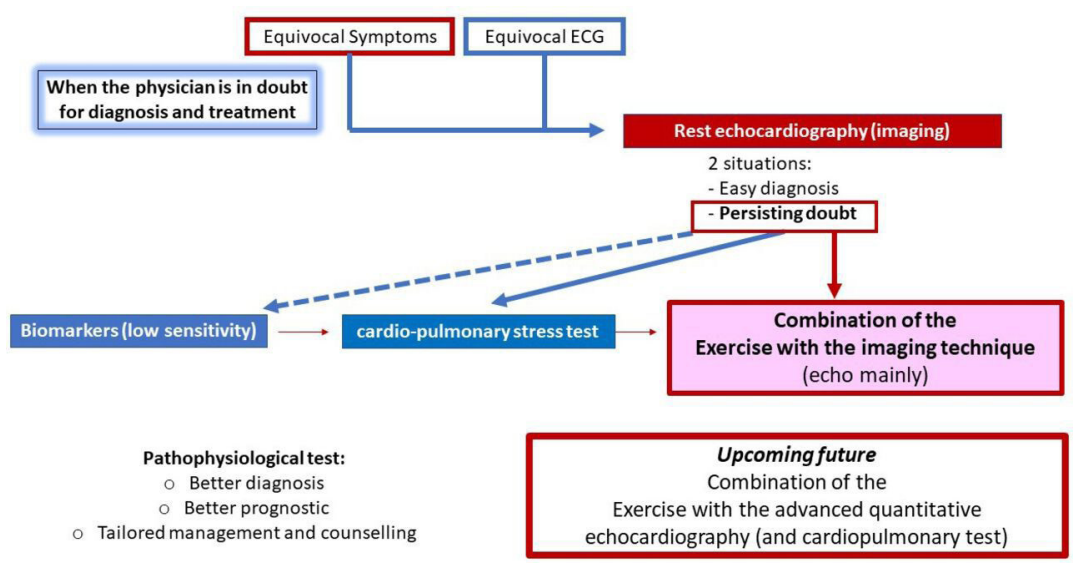

Figure 2 Algorithm justifying the use of exercise stress echocardiography. 


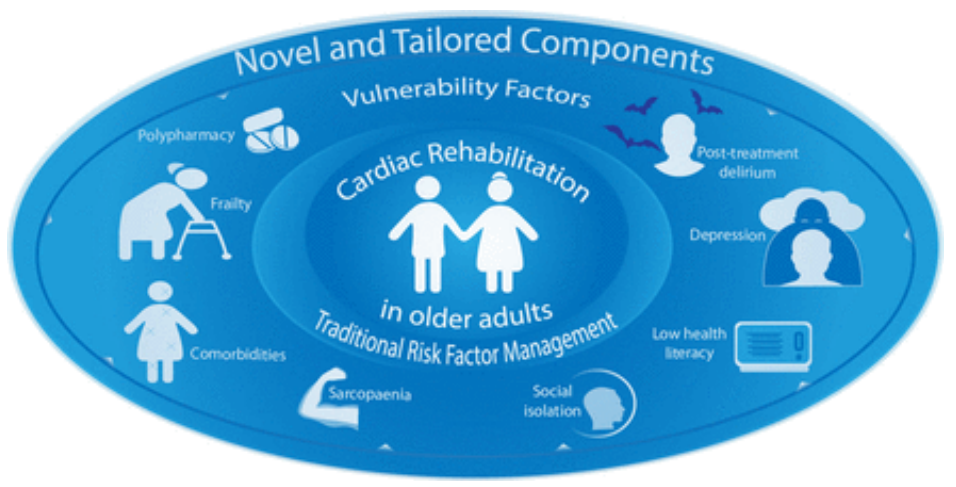

Figure 3 Moving beyond traditional cardiac rehabilitation programmes in multimorbid older adults with functional decline by adding novel and tailored components targeting vulnerability factors.

"has over time evolved from a singlecomponent exercise training programme into a comprehensive lifestyle programme targeting traditional risk factors delivered through group-based sessions. However, the setting (centre-based vs home-based, or a combined model) is especially relevant to older adults." They further suggest that cardiac rehabilitation needs to go beyond traditional risk factors to address other issues relevant to each patient that contribute to suboptimal health. (figure 3)

Neopterin, a surrogate marker of plaque inflammation, is a predictor of coronary artery disease risk. In 4130 patients with suspected stable angina pectoris, Dhar and colleagues ${ }^{5}$ examined the association of plasma neopterin levels, stratified by serum lipid levels, with risk of acute myocardial infarction (AMI). The association of neopterin levels with risk of AMI was strongest in patients with a serum low density lipoprotein cholesterol (LDL-C)
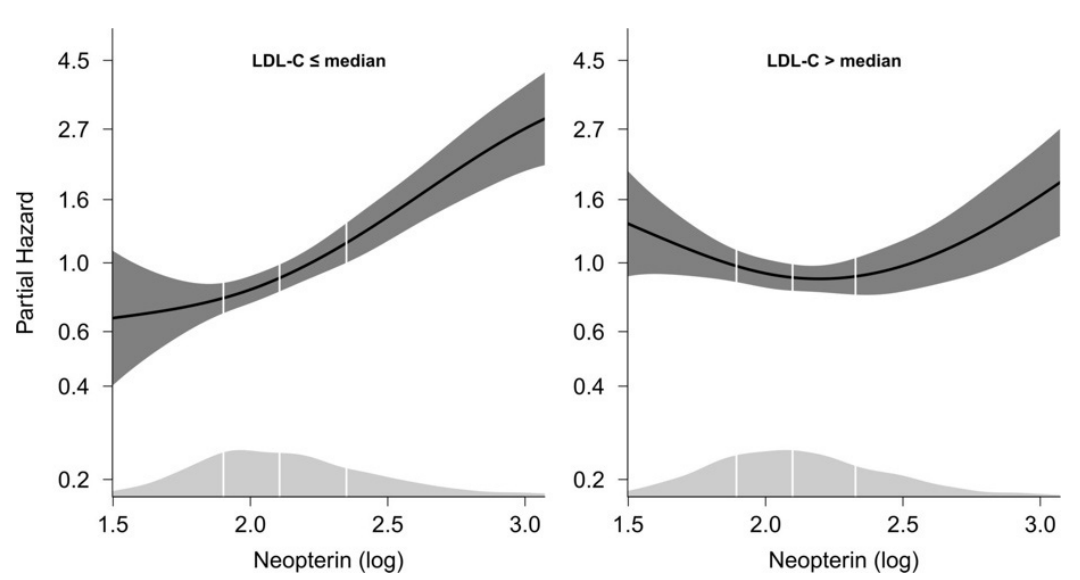

Figure 4 Risk association between log-transformed neopterin and acute myocardial infarction in subgroups of serum LDL-C levels, adjusted for age and sex. Shaded areas around the curves depict $95 \% \mathrm{Cl}$. kernel density plots show the distribution of plasma neopterin. LDL-C, low-density lipoprotein cholesterol.

or an apolipoprotein B100 level below the median.(figure 4) In addition, neopterin levels were strongly associated with risk of AMI in patients with a Vitamin A level above the median, possibly related to the increased production of Vitamin $\mathrm{A}$ in response to oxidised cholesterol metabolites.

In an editorial, ${ }^{6}$ Moss raises the question "What remains unclear with respect to cardiovascular risk modification is whether aggressive reduction of LDL-C is the only target that matters?" Following an elegant and detailed discussion of the issues, he concludes: "While LDL-C accumulation plays an integral role in the formation of atherosclerotic coronary plaque, we now know that there are many more variables that contribute to the acceleration or attenuation of coronary artery disease progression. Is it time for inflammatory biomarkers such as neopterin or hsCRP to be incorporated into 